\title{
Qualitätskriterien forensischer Ambulanzen des Strafvollzugs
}

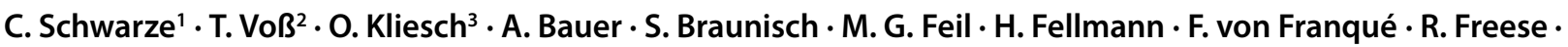 \\ Y. Gretenkord · C. Huchzermeier · V. Jückstock · T. Klemm • H. Kroon-Heinzen · R. Martin · J. Pitzing · K. Wegner · \\ M. Zisterer-Schick
}

Eingegangen: 27. Februar 2018 / Angenommen: 23. April 2018 / Online publiziert: 1. Juni 2018

๑) Der/die Autor(en) 2018. korrigierte Publikation 2019

\section{Zusammenfassung}

Mit dem Gesetz zur Reform der Führungsaufsicht 2007 wurde forensische Nachsorge sowohl für ehemalige Patienten aus dem Maßregelvollzug als auch für Straffällige aus dem Justizvollzug verbindlich etabliert. Im Lauf der vergangenen zehn Jahre entstanden somit bundesweit forensische Ambulanzen des Strafvollzugs, welche den gesetzlichen Auftrag zu Behandlung und Betreuung entlassener Straffälliger länderspezifisch umsetzen. Hierbei handelt es sich teils um neu gegründete Ambulanzen, teils um bereits lange in der Behandlung von straffälligen Menschen tätige Einrichtungen, die ihr bisheriges Angebot um die Betreuung von Menschen unter Führungsaufsicht erweiterten. Diese heterogenen Ambulanzen haben sich seit einigen Jahren in einem jährlich stattfindenden fachlichen Austausch bundesweit vernetzt und Gemeinsamkeiten, Unterschiede und Besonderheiten in der Umsetzung des gesetzlichen Auftrags diskutiert. Angestoßen von der Diskussion um Mindeststandards in forensisch-psychiatrischen Nachsorgeambulanzen des Maßregelvollzugs 2014 entwickelte diese Bundesarbeitsgemeinschaft der forensischen Ambulanzen des Strafvollzug Qualitätskriterien, die trotz verschiedener Länderund Trägerspezifika gemeinsame inhaltliche und formale Parameter erfolgreicher ambulanter Behandlung zur Deliktprävention fokussieren. Der Artikel stellt das Ergebnis dieses Diskussionsprozesses mit den verabschiedeten Qualitätskriterien in den Kategorien Struktur-, Prozess- und Ergebnisqualität vor.

Schlüsselwörter Reform Führungsaufsicht · Forensische Nachsorge · Kriminaltherapie · Forensische Psychotherapie · Qualitätsmanagement

C. Schwarze

claudia.schwarze@stadtmission-nuernberg.de

$\triangle$ Dr. med. T. Voß

tatjana.voss@charite.de

O. Kliesch

oliver.kliesch@vitos-haina.de

1 Psychotherapeutische Fachambulanz, Pirckheimerstraße 16a, 90408 Nürnberg, Deutschland

2 Forensisch Therapeutische Ambulanz, Institut für Forensische Psychiatrie, Charite - Universitätsmedizin Berlin, Seidelstraße 38, 14050 Berlin, Deutschland

3 Vitos forensisch-psychiatrische Ambulanz Hessen, Landgraf-Philipp-Platz 3, 35114 Haina (Kloster),

Deutschland 


\title{
Quality criteria of forensic aftercare treatment services for prison inmates
}

\begin{abstract}
In 2007 the legal reform concerning the supervision of conduct established forensic aftercare as mandatory for patients released from forensic commitment hospitals and offenders released from penitentiaries. Therefore, in the past 10 years forensic outpatient departments have evolved all over Germany in a state-specific manner according to the legal mandate of treating and managing released offenders. Some of these outpatient departments were newly founded and some were long established units for treating and managing offenders that expanded their services for offenders under supervision of conduct. During the past years these heterogeneous outpatient departments have formed a federal network targeting professional exchange, commonalities, discrepancies and distinct characteristics in realizing the legal mandate. Following the debate on minimum requirements in forensic psychiatric aftercare departments of forensic commitment hospitals in 2014, this federal network developed quality criteria. Despite diverse state and trusteeship-specific conditions, these quality criteria emphasize common content and formal factors for a successful forensic outpatient treatment. This article presents the result of a discussion process along with the agreed quality criteria in the categories of the quality of structure, process and results.
\end{abstract}

Keywords Legal reform of supervision of conduct - Forensic aftercare services - Criminal therapy · Forensic psychotherapy · Quality management

Mit dem „Gesetz zur Reform der Führungsaufsicht und zur Änderung der Vorschriften über die nachträgliche Sicherungsverwahrung“" (BGBl, Teil I, S. 513) vom 13.04.2007 wurden erstmals forensische Ambulanzen in das Instrumentarium der Führungsaufsicht einbezogen. In forensischen Ambulanzen sollen straffällig gewordene Menschen nach ihrer Entlassung aus dem Maßregel- oder Strafvollzug (weiter-)behandelt und betreut werden (Sauter et al. 2015). Es entstanden in der Folge Einrichtungen, die diese besondere Form von Betreuung und Behandlung straffälliger Menschen fachgerecht und in enger Kooperation mit Gerichten, der Bewährungshilfe, Stellen der polizeilichen Überprüfung und Überwachung (z.B. Haftentlassenen-Auskunftsdatei Sexualstraftäter - HEADS) und anderen psychosozialen Einrichtungen umsetzen. Die Arbeit der forensischen Ambulanzen findet im Kontext von Bewährungs- und Führungsaufsicht statt. Den Therapeuten obliegt somit ein Doppelmandat von Behandlung und Kontrolle.

Die konkrete Umsetzung des Bundesgesetzes erfolgte eigenständig in den jeweiligen Bundesländern und unter Berücksichtigung lokaler Gegebenheiten. Die Heterogenität der forensischen Ambulanzen des Strafvollzugs zeigt sich bereits in der Suche nach einem Überbegriff, der die Betreuung und Behandlung der Probanden ${ }^{1}$ am treffendsten charakterisiert. Von einigen Ambulanzen wird der Begriff forensische Psychotherapie bevorzugt. Hierdurch soll

\footnotetext{
1 Alternativ werden die Bezeichnungen Klient oder Patient verwendet, die jeweils mit einer unterschiedlichen Bedeutung konnotiert sind. Auch hier gibt es keine einheitliche Bezeichnung über alle Ambulanzen hinweg.

Für die bessere Lesbarkeit wird außerdem durchgehend die männliche Schreibweise verwendet. Die Inhalte beziehen sich jedoch jeweils auch auf weibliche Personen.
}

die Anwendung von psychotherapeutischen Methoden zur Behandlung von (Krankheits-)Symptomen betont werden, die mit erneuter Straffälligkeit assoziiert sind. Anderen erscheint dieser Begriff zu eng an psychische Erkrankungen geknüpft, weshalb sie den Begriff Kriminaltherapie als zutreffender bezeichnen. Hiermit soll betont werden, dass eine Behandlung von straffällig gewordenen Personen eben auch jenseits von psychischen Erkrankungen stattfindet, wobei die Behandlung auf Risikodispositionen abzielt. Dabei können auch andere Interventionen eingesetzt werden, bei denen es sich nicht genuin um psychotherapeutische Methoden handelt (Schwanengel und Endres 2016). Der Begriff Kriminaltherapie wurde wiederum deshalb kritisiert, weil er an einen rechtlichen Status (nämlich Kriminalität, wie sie durch das Strafgesetzbuch definiert ist) anknüpft und damit vernachlässigt, dass in vielen Ambulanzen Angebote für Menschen ohne Verurteilung, aber mit einem Risiko für fremdgefährdende Verhaltensweisen gemacht werden. Auch der Begriff des Risikomanagements wird verwandt, der nach einer Definition von Hart et al. (2003) die Aspekte Überwachung bzw. Risikomonitoring, Therapie, Kontrolle und Opferschutz umfasst. Befürworter dieses Begriffs möchten vor allem darauf hinweisen, dass forensische Ambulanzen Teil koordinierter Maßnahmen in Kooperation mit weiteren externen Institutionen sind. Kritisch wurde diesbezüglich eingewandt, dass sich der Begriff des Risikomanagements eher auf das Zusammenspiel aller beteiligten Institutionen beziehe und damit das Spezifische der forensischen Ambulanzen nicht ausreichend abgebildet werde. Schließlich wird noch der Begriff forensische Rehabilitation genutzt, der sich als erzieherischer, problemlösender und sich wiederholender Prozess mit straffällig gewordenen Menschen definieren lässt (Wade und De Jong 2000). 
Vereinfacht formuliert, wird mit diesem Begriff die aktive Unterstützung von Personen bezeichnet, die nicht mehr straffällig werden wollen oder sollen (Ward und Maruna 2007). Diese Bezeichnung wurde aufgrund des pädagogischen Fokus kritisiert. Darüber hinaus würde auch dieser Begriff die Behandlungsangebote für Menschen ohne Verurteilung, aber mit einem Risiko für fremdgefährdende Verhaltensweisen in vielen Ambulanzen ignorieren. Um einen Kompromiss zwischen diesen Positionen zu finden, wird im nachfolgenden Text von forensisch-therapeutischer Behandlung gesprochen. Hierunter sollen alle therapeutischen - also psychotherapeutischen, psychiatrischen, sozialtherapeutischen und pädagogischen - Interventionen zur Reduktion von veränderbaren Risikofaktoren fallen, die mit erneuter Delinquenz statistisch assoziiert sind. Die Interventionen können sich dabei sowohl auf personen- als auch auf situationsgebundene Merkmale beziehen. Sofern auch andere Instanzen, wie z.B. die Bewährungshilfe, durch rechtliche Vorgaben mit dem Probanden befasst sind, ist eine forensisch-therapeutische Behandlung immer eingebettet in ein institutionelles Geflecht und damit ein Teil eines umfassenden Risikomanagements.

Eine besondere Herausforderung der forensisch-therapeutischen Behandlung liegt in dem Doppelmandat der Ambulanzen: In ihrer deliktpräventiven Aufgabe sollen sie einerseits unterstützend für den Probanden da sein und andererseits Risikoentwicklungen bei dem Probanden juristischen Stellen mitteilen. Auch mit der eingeschränkten Schweigepflicht im Rahmen der Führungsaufsicht bleibt die Abwägung, wann die unter Vertraulichkeit erhaltenen Informationen offenbart werden, eine schwierige Aufgabe für die Behandler. Es wird nicht nur geprüft, ob die Weitergabe risikorelevanten Verhaltens oder eines Weisungsverstoßes die Wahrscheinlichkeit eines Deliktes senkt (weil entsprechende Sicherungsmaßnahmen ergriffen werden können) oder erhöht (weil man von dem Probanden zukünftig keine Informationen mehr bekommt und riskante Entwicklungen somit nicht erfährt), sondern letztlich werden darüber hinaus auch immer die potenziellen Folgen für die Einrichtung in die Entscheidung einbezogen. Dieses Doppelmandat sowie die Tatsache, dass bei jedem Probanden - je nach Rechtsgrundlage - anders mit der Schweigepflicht umgegangen werden muss, ist eine Erschwernis für jeden einzelnen Mitarbeitenden einer forensischen Ambulanz, die es so in keinem anderen therapeutischen Setting gibt.

\section{Forensische Ambulanzen des Strafvollzugs im Bundesgebiet}

Es existieren nur wenige forensische Ambulanzen im Bundesgebiet, die sowohl Maßregel- als auch Strafvollzugsprobanden in ungefähr gleicher Anzahl betreuen. Die überwie- gende Mehrheit ist auf eine der beiden Probandengruppen spezialisiert. Es gibt aktuell in fast allen Bundesländern (Ausnahme Saarland und Niedersachsen) eine institutionalisierte Form von ambulanter Behandlung spezifischer (z. B. mit Sexualstraftaten), aus dem Strafvollzug entlassener Personen.

Der Unterschied zwischen forensisch-psychiatrischen Ambulanzen des Maßregelvollzugs und forensischen Ambulanzen des Strafvollzugs ist hinsichtlich Klientel (Diagnosen, Medikation, Vorbehandlung, Prognose), professioneller Kooperationspartner und rechtlicher Reaktionsmöglichkeiten z.T. gravierend (Schwarze und Freese 2014). Aus diesem Grund sind die bereits veröffentlichten Qualitätskriterien für forensisch-psychiatrische Nachsorgeambulanzen (Freese und Schmidt-Quernheim 2014) zwar ein hilfreicher Ausgangspunkt, jedoch nicht unmittelbar auf die Ambulanzen des Strafvollzugs übertragbar. Des Weiteren wurde deutlich, dass sich die forensischen Ambulanzen des Strafvollzugs in Trägerschaft, Finanzierung, Konzeption, Organisation und personeller Ausstattung sehr unterscheiden.

Aufgrund dessen entstand 2015 eine Arbeitsgruppe (AG) zur Entwicklung von Qualitätskriterien für die deliktpräventive Arbeit mit Personen aus dem Strafvollzug bzw. aus der Sicherungsverwahrung. Mit 16 Vertretern der Bundesländer Baden-Württemberg ${ }^{2}$, Bayern ${ }^{3}$, Berlin $^{4}$, Brandenburg 5 , Hamburg ${ }^{6}$, Hessen ${ }^{7}$, Mecklenburg-Vorpommern ${ }^{8}$, Rheinland-Pfalz ${ }^{9}$, Sachsen ${ }^{10}$, Sachsen-Anhalt ${ }^{11}$, Schleswig-Holstein ${ }^{12}$ und Thüringen ${ }^{13}$ wurde der Heterogenität der Ambulanzsituation in Deutschland Rechnung getragen. Die AG arbeitete in Untergruppen zu den Themen Struktur-, Prozess- und Ergebnisqualität und prüfte die Ergebnisse bei drei gemeinsamen Terminen.

\footnotetext{
2 Jürgen Pitzing, Psychotherapeutische Ambulanz Stuttgart.

${ }_{3}$ Markus G. Feil, Psychotherapeutische Fachambulanz München; Claudia Schwarze, Psychotherapeutische Fachambulanz Nürnberg.

4 Tatjana Voß, Forensisch-Therapeutische Ambulanz Berlin.

5 Stefan Braunisch, Therapeutische Fachambulanz der Justiz Potsdam.

${ }^{6}$ Mounira Jabat/Vivian Jückstock/Fritjof von Franqué, Präventionsambulanz des Instituts für Sexualforschung und Forensische Psychiatrie.

7 Roland Freese, Forensisch-psychiatrische Ambulanz Hessen; Oliver Kliesch, Hessische Fachambulanz.

8 Yvonne Gretenkord, Forensische Ambulanz Rostock.

9 Monika Zisterer-Schick, Psychotherapeutische Ambulanz der Justiz Ludwigshafen.

10 Torsten Klemm, ISONA - Institut für Sozialtherapeutische Nachsorge und Resozialisationsforschung e. V. Leipzig.

${ }^{11}$ Hermann Fellmann, Forensische Ambulanz Sachsen-Anhalt.

12 Hanna Kroon-Heinzen/Christian Huchzermeier, Forensische Fachambulanz Kiel; Reimar Martin, Wendepunkt e. V.; Joachim Tein, Justizministerium Schleswig-Holstein; Kay Wegner, Forensische Ambulanz pro familia Schleswig-Holstein.

13 Andreas Bauer, Therapeutische Ambulanz Erfurt.
} 
Die Zwischenergebnisse wurden auf der bundesdeutschen Ambulanztagung 2016 präsentiert und Rückmeldungen von forensischen Praktikern im weiteren Prozess eingearbeitet. Anspruch der AG war es, Gemeinsamkeiten und Unterschiede $\mathrm{zu}$ benennen, gemeinsame Prinzipien der Arbeit transparent und nachvollziehbar zu beschreiben, Notwendigkeiten zu definieren und somit Maßstäbe für die Qualitätssicherung zu setzen.

Gemeinsames Kerngeschäft der forensischen Ambulanzen des Strafvollzugs in den jeweiligen Bundesländern ist die Arbeit mit verurteilten, schuldfähig straffällig gewordenen Personen mit Weisungen im Rahmen der Führungsoder Bewährungsaufsicht. Das Deliktspektrum bezieht sich in der Regel auf Straftaten gegen die sexuelle Selbstbestimmung und weitere schwere Straftaten gegen Leib und Leben. Je nach Ausrichtung der Ambulanz werden zusätzlich auch Probanden aufgenommen,

- die befürchten, eine Sexual- oder Gewaltstraftat zu begehen,

- die ausschließlich im Dunkelfeld eine Straftat begangen haben,

- die häusliche Gewalthandlungen begangen haben,

- die im Maßregelvollzug untergebracht waren,

- die andere Straftaten (z.B. Brandstiftung) begangen haben.

Daneben bestehen z.T. erhebliche Unterschiede hinsichtlich des Anteils der Probanden mit Bewährung/ Führungsaufsicht/aus der Sicherungsverwahrung, der Art der Delikte, des Anteils psychischer Störungen, des Alters, der Straflast (z.B. Anzahl der [allgemeinen und einschlägigen] Vorstrafen, Anteil der Haftzeit an Lebenszeiten) etc. Um die forensischen Ambulanzen vergleichen zu können, soll daher jede Einrichtung ihre Probandenstruktur hinsichtlich der „Risikobelastung“ individuell beschreiben.

Übergeordnetes Ziel einer forensisch-therapeutischen Behandlung von Probanden mit Sexual- und Gewaltstraftaten ist immer die Verringerung des Risikos zukünftiger strafbarer Handlungen. Dabei ist in der konkreten Praxis allerdings weniger relevant, inwieweit solche Verhaltensweisen juristisch auch zu einer Verurteilung führen würden. Zentral ist der Gedanke, durch die Behandlung jedes fremdschädigende Verhalten zu verhindern und damit potenziell Gefährdete, die Gesellschaft insgesamt und letztlich auch die Probanden vor erheblichen Problemen zu schützen. Gemeinsame Grundlage dieser Arbeit ist ein Menschenbild, in dem jedem Individuum grundsätzlich ein Entwicklungspotenzial zugeschrieben wird.

Von dem anfänglichen Ziel, Mindeststandards zur Qualitätssicherung zu entwickeln, ist die AG im Laufe des Prozesses zurückgetreten. Gründe hierfür lagen zum einen in der Heterogenität der Ambulanzen, die eine Einigung erschwerte, und zum anderen in der zum jetzigen Zeitpunkt begrenzten Evidenzbasierung zur Wirksamkeit ambulanter deliktpräventiver Behandlung im deutschsprachigen Raum (Sauter et al. 2017). Stattdessen wurden die im Folgenden aufgeführten Qualitätskriterien, die nach Struktur-, Prozessund Ergebnisqualität ${ }^{14}$ unterschieden werden, im November 2017 verabschiedet. Diese sind als vorläufig zu betrachten und sollten in den nächsten Jahren entsprechend evaluiert und weiterentwickelt werden.

\section{Strukturqualität}

Für eine gute Strukturqualität sind verschiedene Aspekte in der Personal- und Sachausstattung einer Ambulanz wichtig. So soll die Arbeit einer forensischen Ambulanz des Strafvollzugs durch ein multiprofessionelles Team aus psychologischen und sozialpädagogischen und/oder Mitarbeitenden anderer Berufsgruppen mit spezifischer Berufserfahrung bzw. Qualifikation durchgeführt werden (Voß et al. 2015). Die notwendige Qualifikation der Mitarbeitenden (SQK 1) richtet sich dabei nach der inhaltlichen Schwerpunktsetzung und dem Bedarf der zu versorgenden Klientel der jeweiligen Ambulanz. Im Gegensatz zu forensischpsychiatrischen Ambulanzen ist ein Facharzt unter den Mitarbeitenden aufgrund der anderen Probandenstruktur nicht obligatorisch. Die Leistung kann alternativ über ein gutes Kooperationsnetzwerk abgerufen werden. Optimal ist eine gemischtgeschlechtliche Zusammensetzung des Teams.

Das spezifische Wissen zur forensisch-therapeutischen Behandlung (z.B. Risikobeurteilung, Sexualtherapie, Rechtspsychologie) muss in der Regel während der forensischen Tätigkeit erworben, aufgebaut und weiterentwickelt werden. Die Qualifikation der Mitarbeitenden soll somit über regelmäßige fachspezifische Fort- und Weiterbildung sowie Intervision und Supervision gewährleistet (SQK 11) werden. Für die Durchführung einer psychotherapeutischen Behandlung von deliktrelevanten psychischen Störungen ist eine Weiterbildung in einer wissenschaftlich anerkannten Therapierichtung und/oder eine Approbation als psychologischer oder ärztlicher Psychotherapeut notwendig.

Um die personellen Ressourcen zielgerichtet einzusetzen, bedarf es in jeder Ambulanz einer Verwaltungsstelle zur Gewährleistung der organisatorischen Abläufe. Jede forensische Ambulanz benötigt außerdem eine organisatorische und eine fachliche Leitung (SQK 2), die auch in Personalunion durchgeführt werden kann. Die fachliche Leitung sollte über mehrjährige, fachspezifische Erfahrung sowie die Voraussetzung zur Durchführung von Psychotherapie (s. oben) verfügen. Die Dienst- und Fachaufsicht müssen benannt und in ihren Funktionen klar beschrieben sein. Für

\footnotetext{
${ }^{14}$ Nachfolgend bezeichnet mit SQK Struktur-, PQK Prozess- und EQK Ergebnisqualitätskriterien.
} 
alle Mitarbeitenden sollten Stellen- und Tätigkeitsbeschreibungen vorliegen. Insbesondere wegen der häufig auftretenden Fragen zu Schweige- und Offenbarungspflicht sollte ein juristischer Ansprechpartner für jede forensische Ambulanz benannt sein (SQK 3).

Hinsichtlich der räumlichen Ausstattung ist zu gewährleisten, dass jedem Mitarbeitenden (inkl. Verwaltung) während dessen Arbeitszeit ein eigener Arbeitsplatz zur Verfügung steht (SQK 9). Außerdem sollte eine forensische Ambulanz mit ausreichender Anzahl von schallgeschützten Behandlungsräumen, mindestens einem Raum für Gruppengespräche, Teamsitzungen, runde Tische u. Ä., einem Sozialraum für alle Mitarbeitenden und für Personal und Probanden getrennte Sanitärbereiche ausgestattet sein. Des Weiteren sind Flächen für einen Wartebereich sowie für Archiv, Lager und EDV-Technik notwendig. Um Gefahrensituationen für die Mitarbeitenden zu minimieren, müssen bauliche Maßnahmen, eine entsprechende technische Ausstattung sowie Regelungen in einem Sicherheitskonzept getroffen werden. Bei aufsuchender Arbeit sollten ein Dienstfahrzeug und ein Mobiltelefon zur Verfügung gestellt werden. Daneben ist ein ausreichender Etat für Sachmittel wie Fachbücher, Diagnostika, Fortbildungen etc. notwendig. Die Personal- und Sachkosten der forensischen Ambulanz sollten langfristig und ausreichend gesichert sein, um die in der Regel mehrjährig notwendige forensische Arbeit nachhaltig sicherstellen zu können. Zur effizienten Strukturierung des Tagesgeschäftes (elektronische Probandenakte) und zu Evaluationszwecken (Jahresstatistik, Verlaufsmessung) wird ein IT-gestütztes Probandendokumentationssystem empfohlen (SQK 11).

Entsprechend des „Risk“-Prinzips (Andrews und Bonta 2010) erfordern Probanden mit einem hohen Rückfallrisiko mehr personelle Ressourcen als Probanden mit einem niedrigen Rückfallrisiko. Die Probandenfallzahl pro Vollzeitstelle (,caseload“) als Maß der Auslastung einer Ambulanz hängt somit von Parametern wie der Risikobelastung der versorgten Probanden, der Kontaktfrequenz, der Kontaktart (empfangend/aufsuchend, Einzel-/Gruppentherapie) und der jeweiligen Behandlungsangebote (Psychotherapie ohne/mit hohem Anteil an Soziotherapie, Risikobeurteilung ohne Psychotherapie, Ersttherapie/Nachsorge) ab (SQK 4). Trotz der Heterogenität der bundesweiten forensischen Ambulanzen des Strafvollzugs ergab eine anonymisierte Befragung der Leitungen unter Berücksichtigung eines typischen „case mix“ ihrer jeweiligen Ambulanz ein Verhältnis von Vollzeitkraft zu Probandenanzahl von 1:18. Unter diesen Bedingungen ist nach Expertenmeinung eine den Qualitätskriterien entsprechende Arbeit mit Probanden aus dem Strafvollzug möglich.

Als ein weiteres Maß für die Auslastung einer Ambulanz sollte die Anzahl der Stunden im geplanten, direkten Probandenkontakt pro Vollzeitstelle herangezogen werden.
Auch dieses Maß ist von den oben genannten Parametern abhängig. Die Arbeitsgruppe empfiehlt in Anlehnung an die Erhebung der KrimZ (Gregório Hertz et al. 2017) als Richtlinie, etwa ein Drittel der Arbeitszeit mit geplantem, direktem Probandenkontakt zu verbringen. Ein weiteres Drittel wird durch indirekte Arbeit am Probanden wie Aktenstudium, Vor-/Nachbereitung, Behandlungsplanung, Supervision/Fallbesprechungen, runde Tische, Dokumentation etc. ausgefüllt. Das verbleibende Drittel wird für Qualifikationsmaßnahmen, Aufbau und Erhalt von Kooperationsstrukturen u. Ä. aufgewandt. Die Leitungsanteile sind gesondert auszuweisen und fallen nicht mit in die Berechnung für die Auslastung einer Ambulanz.

Für die forensisch-therapeutische Behandlung soll ein schriftlich ausgearbeitetes Behandlungskonzept vorliegen, welches leitlinienorientiertes Arbeiten ermöglicht (SQK 10). Zur Sicherung der Prozess- und Ergebnisqualität sind eine interne und externe Evaluation sowie die Sicherstellung deren Finanzierung notwendig (SQK 11). Eine Behandlung soll auch dann fortgeführt werden - fachliche Notwendigkeit und Einverständnis des Probanden vorausgesetzt -, wenn die Bewährungs- oder Führungsaufsicht ausläuft (SQK 6). Auch Probanden, die sich nach abgeschlossener Behandlung in schwierigen Lebenssituationen erneut an die forensische Ambulanz wenden, werden zur Deliktprävention wieder aufgenommen.

Für eine planbare Probandenversorgung soll die regionale Zuständigkeit der forensischen Ambulanz klar festgelegt sein (SQK 8). Die Einrichtung sollte mit öffentlichen Verkehrsmitteln und innerhalb einer zumutbaren Anreisezeit erreichbar sowie die Finanzierung der Fahrtkosten verbindlich geregelt sein. Die Entfernungen müssen bei aufsuchendem Arbeiten auch für die Mitarbeitenden zumutbar und effizient sein.

Die forensischen Ambulanzen des Strafvollzugs halten keine gesonderten Bereitschaftsdienste vor (SQK 5). Die Bearbeitung aktueller Krisen erfolgt ausschließlich während der jeweiligen Geschäftszeiten. Es sollte jedoch ein Krisen- und Risikomanagement in Form von individualisierten Notfallplänen mit vorab geregelten Schweigepflichtentbindungen vorliegen.

Für eine bedarfsgerechte, ambulante Arbeit mit straffälligen Menschen ist die Zusammenarbeit mit anderen Einrichtungen erforderlich (SQK 7). Um den vielfältigen Anforderungen im Spannungsfeld von Hilfe und Kontrolle in der deliktpräventiven Arbeit gerecht zu werden, müssen entsprechende Netzwerke aufgebaut und gepflegt werden. Neben zuweisenden Institutionen wie z. B. Justizvollzugsanstalten, handelt es sich einerseits um extramurale Helfernetzwerke wie z. B. Suchtberatungsstellen, sozialpsychiatrische Dienste, betreutes Wohnen und Fachärzte, andererseits um Netzwerke der Kontrolle wie z. B. polizeiliche Überwachungsinstitutionen und Gerichte. Eine Sonderstellung neh- 

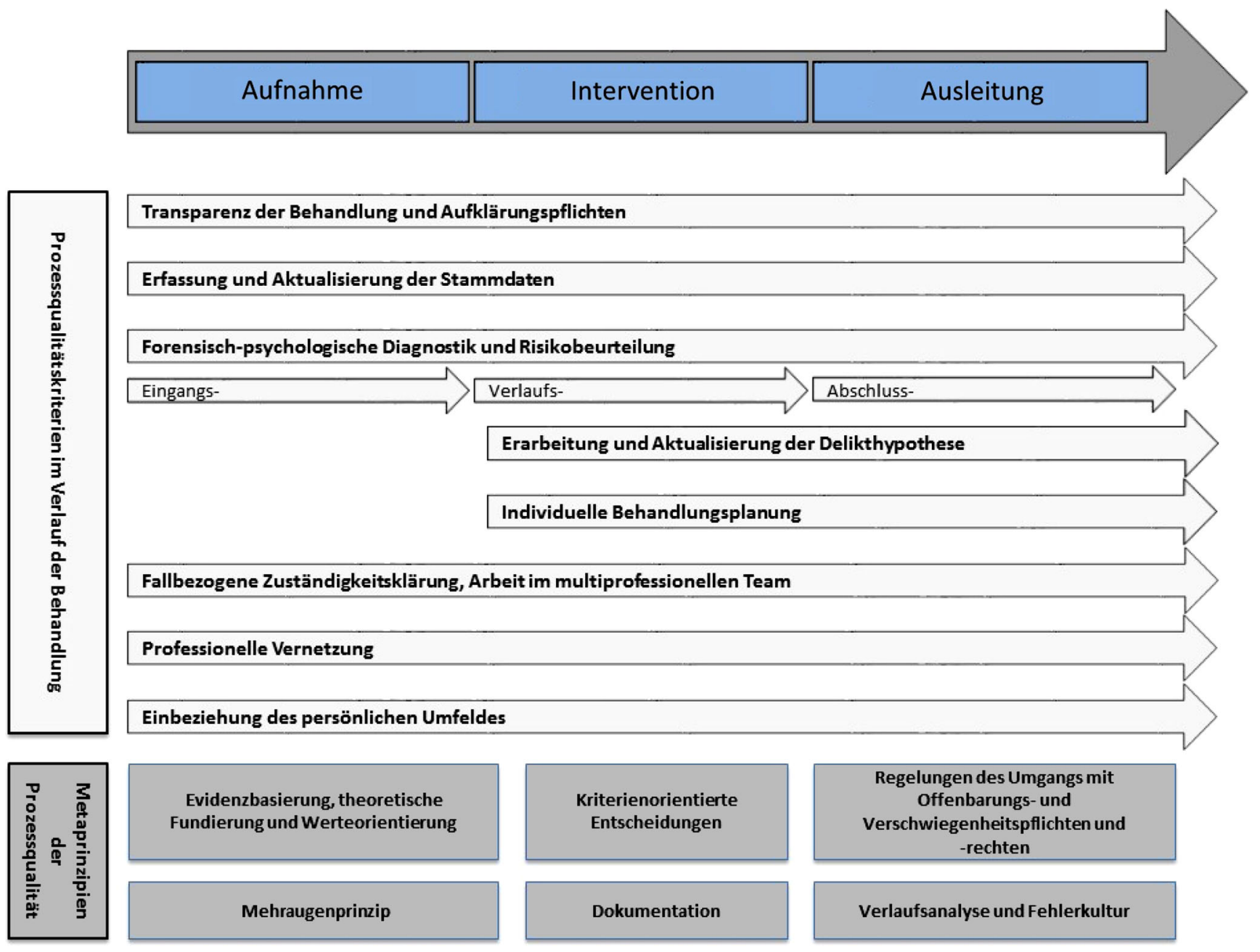

Abb. 1 Kriterien und Metaprinzipien zur Prozessqualität

men die Bewährungshilfe und in einigen Bundesländern die Führungsaufsichtsstellen bzw. die dortigen sozialen Dienste ein, die ggf. in allen drei oben genannten Rollen (Zuweisung, Hilfe, Kontrolle) tätig sein können und schon nach dem Gesetz engste Partner der forensischen Ambulanzen sind.

\section{Prozessqualität}

Die Kriterien für die inhaltliche Arbeit der forensischen Ambulanzen des Strafvollzugs werden in Qualitätskriterien im Verlauf der Behandlung und sog. Metaprinzipien der Prozessqualität unterschieden. In Abb. 1 sind diese einem beispielhaften Ablauf einer forensisch-therapeutische Behandlung mit Unterteilung in Aufnahme-, Interventionsund Ausleitungsphase zugeordnet. Die genaue Zeitdauer und Methodik in den einzelnen Phasen orientieren sich am Einzelfall.
Im Rahmen des Aufnahmeverfahrens erfasst die forensische Ambulanz die Stammdaten des Probanden (PQK 1.2). Dazu zählen: Aufnahmedatum, Beginn der Behandlung, Kontaktdaten des Probanden, der entlassenden JVA und der Kooperationspartner, Anlassdelikt, Vorstrafen, Gesetzesgrundlage der Behandlung (Führungsaufsicht, Bewährung, Vorstellungs- und/oder Therapieweisung), voraussichtliches Ende der Bewährung/Führungsaufsicht, Diagnosen gemäß aktueller Ausgabe des ICD oder DSM, Art der Medikation. Außerdem werden Anzahl und Art der Kontakte mit der Ambulanz sowie Fehlzeiten dokumentiert.

Bereits im Erstgespräch muss die Aufklärung des Probanden in Bezug auf rechtliche und institutionelle Regelungen erfolgen und dokumentiert werden (PKQ 1.1). Dazu gehört eine transparente Aufklärung über die Rahmenbedingungen der forensisch-therapeutischen Behandlung (z.B. in einer Behandlungsvereinbarung), konkret über Datenschutz, Offenbarung- und Verschwiegenheitspflichten bzw. -rechte, juristische Grundlagen der Behandlung und mögliche Nebenwirkungen der Therapie. 
Im Rahmen des Aufnahmeverfahrens findet eine forensisch-psychologische Diagnostik und Risikobeurteilung statt, aus der eine individuelle Rückfallprognose und Zielbereiche für Interventionen abgeleitet werden (PQK 1.3). Dabei werden psychische Störungen (insbesondere Persönlichkeitsstörungen), ggf. eine psychiatrische Diagnostik, mit Selbst- und Fremdbeurteilungsverfahren erhobene situative und personale Risikofaktoren sowie personale und soziale Ressourcen und Schutzfaktoren berücksichtigt.

Die forensisch-psychologische Diagnostik basiert zum einen auf dem persönlichen Kontakt mit dem Probanden, sowohl in der Einrichtung als auch nach Möglichkeit im häuslichen Umfeld, und zum anderen auf der Sichtung juristischer und weiterer relevanter Unterlagen. Hierfür sollten Urteile, Bewährungs- bzw. Führungsaufsichtsbeschlüsse, ein Bundeszentralregisterauszug, Gutachten, ggf. Fremdanamnesen und Stellungnahmen der entlassenden Einrichtungen eingeholt werden.

Die Ergebnisse von Diagnostik und Risikobeurteilung sind mindestens einmal jährlich zu überprüfen, zu ergänzen und aktualisiert in die Behandlung einzubeziehen. Zusätzlich dazu soll eine systematische Überprüfung der diagnostisch-prognostischen Einschätzung bei besonderen Anlässen, wie Hinweisen auf verringertes psychosoziales Funktionsniveau, akutem Rückfallrisiko, stattgefundenem Rückfall oder sonstigen rückfall- oder krisenrelevanten Informationen, erfolgen.

Ausgangspunkt für die Behandlungsplanung und die therapeutische Arbeit mit straffällig gewordenen Menschen ist eine individuelle Delikthypothese (PQK 1.4). Diese enthält die konkreten personalen und situativen Faktoren, die zum Anlassdelikt des Probanden beigetragen haben. Insbesondere nach der Haftentlassung sind zahlreiche Änderungen an diesem Faktorengefüge denkbar. Daher sollen die aktuellen und realen Lebensumstände fortlaufend einbezogen werden und zu einem zukünftigen Risikoentstehungsmodell weiterentwickelt werden.

Mit einer Behandlungsplanung wird die individuelle Fallarbeit beschrieben und damit sowohl für Therapeuten, kooperierende Einrichtungen und auch für die Probanden selbst transparent gemacht (PQK 1.5). Die Behandlungsintensität soll dabei dem Rückfallrisiko („Risk“-Prinzip; Andrews und Bonta 2010) angepasst werden. Die Planung soll mindestens konkrete Behandlungsziele (z.B. Verbesserung der Impulskontrolle, Reduzierung devianter Fantasietätigkeit, Aufbau prosozialer Kontakte), Interventionen (z.B. Psychotherapie, Risiko-Monitoring, sozialtherapeutische Begleitung, Medikation) und Settings (z. B. Frequenz, Einzel-/Gruppentherapie) enthalten. Der Behandlungsplan soll mindestens einmal jährlich sowie bei aktuellem Anlass fortgeschrieben werden. Dabei ist auf die zuletzt festgelegten Behandlungsziele und die ggf. aktualisierte
Delikthypothese Bezug zu nehmen. Veränderungen sind zu beschreiben und zu bewerten.

Als ein weiteres Qualitätskriterium gilt die Einbeziehung des persönlichen Umfelds des Probanden in die Behandlung (PQK 1.8). Auf diese Weise wird ermöglicht, die im Gespräch gewonnenen Erkenntnisse anhand der häuslichen bzw. tatsächlichen Lebensrealität zu überprüfen und die Perspektiven anderer Beteiligter einzubeziehen. Prospektiv betrachtet können sich Interventionen im Umfeld als wirksamer herausstellen als die ausschließliche Fokussierung auf den Indexprobanden, z. B. bei der Gewährleistung des Kinderschutzes bei Menschen mit pädosexuellen Delikten. In welcher Form das persönliche Umfeld einbezogen wird (z.B. Angehörigenarbeit, Hausbesuche, Kooperation mit Jugendamt oder Polizei), bleibt eine Einzelfallentscheidung und erfolgt gemäß gesetzlicher Vorgaben und mit Einverständnis aller Beteiligten.

Die forensisch-therapeutische Behandlung erfordert ein ganzheitliches Fallverständnis aus den Perspektiven unterschiedlicher Professionen. Damit therapeutische Prozesse im multiprofessionellen Team arbeitsteilig organisiert werden können, ist die interne Fallverantwortung zu dokumentieren (PQK 1.6). Es erfolgen eine Aufgabenteilung der insgesamt notwendigen Arbeitsschritte auf die beteiligten Berufsgruppen und die Zusammenführung der jeweiligen Arbeitsergebnisse in Form von Besprechungen.

Um eine doppelte Aufgabenübernahme zu vermeiden, ist auch zwischen der forensischen Ambulanz und den kooperierenden Personen eine klare Absprache notwendig (PQK 1.7). Hierfür sollen zum einen die beteiligten Personen oder Institutionen konkret benannt werden (z. B. JVA, Bewährungshilfen, Gerichte, Führungsaufsichtsaufstellen, Polizei, Wohnheime, niedergelassene Therapeuten und Ärzte). Zum anderen sollen die geklärte Zuständigkeit dokumentiert und die Informationswege (z.B. regelmäBige Fallkonferenzen) und -anlässe (z.B. bei riskanten Entwicklungen oder Weisungsverstößen) transparent definiert werden. Berichte und kollegialer Austausch erfolgen stets unter Berücksichtigung der Grundsätze der gesetzlichen Offenbarungs- und Verschwiegenheitspflichten bzw. -rechte.

Grundsätzlich soll eine forensisch-therapeutische Behandlung evidenzbasiert bzw. theoriegeleitet sowie an ethischen Kriterien orientiert sein (PQK 2.1). Interventionen sollen sich daher an wissenschaftlich fundierten Rehabilitationsmodellen orientieren. Es seien an dieser Stelle das Risk-Need-Responsivity Model (Andrews und Bonta 2010) und/oder das Good Lives Model (Ward und Gannon 2006) genannt, die eine einzelfallbezogene Risiko- und Bedarfsbeurteilung zum Zweck der Behandlungsplanung ermöglichen. Diese soll unter Zuhilfenahme einschlägiger (klinisch-)psychologischer Testverfahren und evaluierter Prognoseinstrumente vorgenommen werden. Um Aus- 
sagen zum Behandlungsverlauf treffen zu können, sind entsprechende Messungen mindestens vor, während und mit Abschluss einer Behandlung notwendig.

Die individuelle Behandlungsplanung sowie fallbezogene Entscheidungen über Maßnahmen und Interventionen sollen nachvollziehbar begründet, kommuniziert und dokumentiert werden (PQK 2.2). Kriterien und Parameter für die Wahl des Behandlungssettings, der Interventionen und behandlerische Entscheidungen sollen in den Konzeptionen der jeweiligen Ambulanzen als Rahmenbedingungen schriftlich festgelegt sein. Zugleich werden auch die Grenzen und notwendigen Rahmenbedingungen der Behandlung festgehalten.

Probanden und weitere Beteiligte müssen von Beginn an über die geltenden gesetzlichen Bestimmungen zur Offenbarung und Verschwiegenheit transparent informiert werden (PQK 2.3). Je nach rechtlichem Status des Probanden - freiwillige Behandlung, Bewährungs- oder Führungsaufsicht - unterscheiden sich diese in wesentlichen Aspekten. Eine Konkretisierung des Vorgehens, z. B. für das Berichtsund Konferenzwesen mit Bewährungshilfe und Führungsaufsichtsstelle oder für Ausnahmesituationen wie Krisen, soll entsprechend sowohl konzeptionell für die Einrichtung als auch individuell mit dem Probanden festgelegt sein.

Jeder individuelle Behandlungsverlauf eines Probanden soll regelmäßig analysiert und das Ergebnis dokumentiert werden (PQK 2.6), um die Qualitätssicherung und eine fachgerechte Kommunikation ambulanzintern sowie auch extern gegenüber Kooperationsinstitutionen und Auftraggebern zu gewährleisten. Hierfür werden u.a. Krisen, delinquenzrelevantes Verhalten und Weisungsverstöße erfasst. Eine umfassende Analyse bezieht sowohl Kriterien für erfolgreiche Verläufe ein, z.B. erreichte Ziele, Entschärfung von Risikofaktoren oder verbesserte Ansprechbarkeit, wie auch die Analyse möglicher Fehler oder unzureichender Ressourcen, um für die zukünftige Arbeit notwendige Änderungen ableiten zu können.

Als eine Methode der Qualitätssicherung und Fehlerminimierung sollte das Mehraugenprinzip (PQK 2.4) angewandt werden. Es dient der Absicherung durch Redundanz und der Verbesserung der Ergebnisse durch die Erfassung verschiedener Sichtweisen, v. a. auch unterschiedlicher Professionen. Entsprechend bedarf es regelmäßiger Fall- und Teambesprechungen, externer Supervision sowie der Abstimmung mit den im Einzelfall verbundenen Kooperationspartnern.

Die Dokumentation relevanter interner und externer Informationen zum Behandlungsprozess soll nachvollziehbar, regelhaft, fachgerecht und zeitnah erfolgen (PQK 2.5). Die Dokumentation ist einrichtungsintern standardisiert. Sie beinhaltet Stammdaten, fallvorbereitende Informationen (inkl. Entscheidungen über Maßnahmen und Interventionen) sowie direkte und indirekte Leistungen für den Probanden und wird in geeigneter Form (konventionelle oder elektronische Akte) erfasst. Angaben in den Akten sind aktuell zu halten und mit dem jeweiligen Datum zu versehen. Die Vernichtung der Akten wird ordnungsgemäß nach den gesetzlichen Vorgaben vorgenommen.

\section{Ergebnisqualität}

Eine forensisch-therapeutische Behandlung soll (erneute) Gewalttaten verhindern. Unter Gewalttaten werden hier dabei folgende Aspekte verstanden:

- Gewalt i.Allg.: jeder tatsächlich ausgeübte, versuchte oder angedrohte physische Schaden an anderen Personen (Webster et al. 1997);

- häusliche Gewalt: jeder tatsächliche, versuchte oder angedrohte physische Schaden, an einer Person, mit der eine intime sexuelle Beziehung geführt wird oder wurde (Kropp et al. 1999);

- sexuelle Gewalt: jeder tatsächliche, angedrohte oder versuchte sexuelle Kontakt mit einer Person, die hiermit nicht einverstanden ist oder sich nicht einverstanden erklären kann (Hart et al. 2003). Hierunter ist außerdem die Nutzung und/oder Verbreitung von Abbildungen zu verstehen, die sexuelle Gewalt zum Nachteil von Kindern beinhalten oder diese in sexuellen Posen zeigen;

- Stalking: jede(r) unerwünschte(r) und wiederholte(r) Mitteilung, Kontakt oder andere Verhaltensweise, die bei Betroffenen absichtlich oder fahrlässig zu nachvollziehbaren Ängsten oder Sorgen um die eigene Sicherheit oder um die Sicherheit ihnen Bekannter führt (Kropp et al. 2008).

Die Interventionen zielen somit darauf ab, kriminogene Persönlichkeitsmerkmale zu modifizieren, die Ansprechbarkeit für Interventionen zu erhöhen und Verhaltensänderungen des Probanden anzustoßen und ggf. einzuüben. Um den Erfolg der forensisch-therapeutischen Behandlung zu überprüfen, sollte jede Ambulanz nicht nur direkt deliktbezogene Kriterien erheben und beurteilen, sondern auch Kriterien, die mittelbar mit Delinquenz und Therapieerfolg im Zusammenhang stehen.

Als direkt deliktbezogene Kriterien soll die Gewaltvermeidung (EQK 1.1), die Reduktion von Risiko (EQK 1.2) und die erfolgreiche Anbindung der Probanden an die Ambulanz (EQK 1.3) betrachtet werden.

Eine forensisch-therapeutische Behandlung kann dann als erfolgreich gelten, wenn erneute einschlägige Delikte (EQK 1.1.1) sowie erneute nichteinschlägige Gewalthandlungen, sonstige Delinquenz und weitere dissoziale Verhaltensweisen (EQK 1.1.2) durch die therapeutische Arbeit verhindert wurden. Diese Erfolgskriterien sollten zum einen während der Behandlung und zum anderen nach Ende der 
Behandlung mindestens nach drei, möglichst aber nach fünf Jahren erhoben werden. Neben dem Bundeszentralregisterauszug dienen als weitere wichtige Quellen die klinische Exploration und die Fremdanamnese; in beidem soll explizit nach erneuten Gewalthandlungen gefragt werden. Bei näherer Betrachtung können selbst bei rückfälligen Probanden Aussagen über einen zumindest teilweisen Erfolg der forensisch-therapeutischen Behandlung getroffen werden. Dieser liegt dann vor, wenn im Falle eines Rückfalls die Gewalthandlung gegenüber dem Zeitraum vor der Therapie weniger schwerwiegend war oder wenn die Frequenz, in der Delikte begangen werden, deutlich abgenommen hat.

Eine forensisch-therapeutische Behandlung kann auBerdem als erfolgreich gelten, wenn sich die zu Beginn der Therapie ermittelte Risikokategorie (z.B. hoch, moderat, gering) für erneute, einschlägige Gewalthandlungen (EQK 1.2.1), die gemessenen Risiko-Scores (summarische Zusammenfassung dynamischer Risikofaktoren) für erneute, einschlägige Gewalthandlungen (EQK 1.2.2) oder spezifische Risikofaktoren, die im Rahmen einer strukturiertklinischen Beurteilung als relevant für die Deliktgenese erachtet wurden (EQK 1.2.3), in der abschließenden Beurteilung reduziert haben. Für eine Einschätzung der Reduktion des Risikos sind immer Fremdbeurteilungsverfahren mit statischen und dynamischen Risikofaktoren heranzuziehen. Zur Evaluation ist notwendig, dass die Ausprägung von Risikofaktoren einmal zu Beginn der Behandlung und fortlaufend bis zu deren Ende erfasst wurde. Dabei soll die Begründung für das jeweilige Rating nachvollziehbar festgehalten werden.

Zudem kann eine forensisch-therapeutische Behandlung als erfolgreich gelten, wenn die Probanden im therapeutischen Setting gehalten werden können (EQK 1.3). Ein Therapieabbruch ist im forensischen Kontext allerdings häufiger als in der allgemeinen Psychotherapie und bei psychiatrischen Nachsorgebehandlungen. Als abgebrochen gilt eine Behandlung, wenn eine relevante Veränderung von $\mathrm{Ri}$ sikomerkmalen und/oder dysfunktionalen Verhaltensweisen noch nicht erreicht werden konnte und die Behandlung vonseiten des Probanden nicht fortgesetzt wird. Die forensische Ambulanz soll die Anzahl der Therapieabbrüche erfassen, die von den Probanden ausgehen, und dabei möglichst die genauen Gründe für den Abbruch festhalten. Die Entwicklung der Anzahl der Behandlungsabbrüche soll in Abständen kritisch reflektiert und ggf. sollen Maßnahmen zur Verbesserung des „Therapie-Commitment“" abgeleitet werden.

Als mittelbar deliktbezogene Kriterien, die auf einen Behandlungserfolg hinweisen, sollen Faktoren der therapeutischen Ansprechbarkeit (EQK 2.1) zu Beginn und fortlaufend bis zum Ende der Behandlung nachvollziehbar erfasst werden. Dazu zählen z. B. Feindseligkeit, negative Haltung zur forensischen Ambulanz, niedrige Behandlungsmotivation, geringe Veränderungsbereitschaft sowie kultureller Hin- tergrund (von Franqué und Briken 2017). Wenn diese Faktoren durch geeignete Strategien im Verlauf der Betreuung durch die forensische Ambulanz verbessert werden konnten, so ist dies als Erfolg zu bewerten.

Die Compliance der Probanden (EQK 2.3), d.h. deren aktive, motivierte und zuverlässige Mitarbeit, wird ebenfalls als ein Hinweis auf eine erfolgreiche forensisch-therapeutische Behandlung gewertet. Da sich Compliance nicht direkt messen lässt, muss auf Merkmale zurückgegriffen werden, die Compliance widerspiegeln. Hierzu zählen beispielsweise die Zuverlässigkeit, mit der Probanden ihre Termine einhalten (Verhältnis von wahrgenommenen zu abgesagten bzw. zu unentschuldigt versäumten Terminen), die Einhaltung von juristischen Weisungen und therapeutischen Empfehlungen, die regelmäßige Einnahme von Medikamenten, falls solche verordnet wurden.

Eine ressourcenorientierte, forensisch-therapeutische Behandlung berücksichtigt die Grundbedürfnisse der Probanden. Die Zufriedenheit der Klientel wird in vielen Untersuchungen zur Wirksamkeit von psychotherapeutischer Behandlung erhoben. Ein Qualitätsmerkmal forensischtherapeutischer Behandlung ist es, wenn sich die behandelten Probanden selbst zufrieden über die Behandlung äußern (EQK 2.2.1). Da ein direkter Zusammenhang zwischen subjektiver Zufriedenheit der Probanden und einer tatsächlichen Verbesserung der Lebensumstände bzw. einer Risikominderung nicht belegt ist, sagt dieses Kriterium vermutlich mehr über die Qualität der Beziehung zwischen Proband und Therapeut bzw. der Ambulanz aus. In diesem Sinne kann die Abfrage der subjektiven Zufriedenheit als Berücksichtigung der Probandensicht gewertet werden und hilfreiche Anhaltspunkte für das eigene Auftreten liefern. Ebenso sollten die allgemeine Lebenszufriedenheit und die subjektiv erlebte bzw. psychopathologische Belastung der Probanden (EQK 2.2.2) idealerweise zu Beginn der Behandlung und fortlaufend bis zum Ende erfasst werden. Eine Verbesserung der Werte während der Behandlung deutet auf einen Erfolg der Maßnahme hin.

Straffällig gewordene Menschen leben häufig in sozial randständigen Verhältnissen. Die soziale Integration bzw. die soziale Unterstützung beeinflusst deutlich das Ausmaß gesundheitlicher Beschwerden. Eine forensisch-therapeutische Behandlung war erfolgreich, wenn die Probanden in sozialen Belangen (z. B. sichere Wohn- und Finanzverhältnisse, Integration ins Berufsleben, sinnvolle Freizeitgestaltung) unterstützt wurden und Fortschritte im Vergleich zur Ausgangssituation erzielt haben (EQK 2.4). Da sich soziale Integration nicht direkt messen lässt, muss auf Merkmale zurückgegriffen werden, die soziale Integration widerspiegeln.

Als letztes Kriterium für eine erfolgreiche forensischtherapeutische Behandlung ist die Leistungsfähigkeit einer forensischen Ambulanz zu sehen. Sie hängt wesentlich 
von der Leistungsfähigkeit, Belastbarkeit und Zufriedenheit der Mitarbeitenden sowie der Teamstabilität ab (EQK 2.5). Grundlagen dafür sind in der Struktur- und Prozessqualität enthalten. Messbar sind Kriterien wie befristete/unbefristete Arbeitsverträge, Mitarbeiterfluktuation und Krankenstand. Weiter sind Möglichkeit und Anzahl von Fortbildungen, Qualifizierungsmaßnahmen etc. zähl- und auswertbar. Nicht zuletzt spiegelt ein respektvoller Umgang der Mitarbeitenden und Vorgesetzten untereinander eine Haltung wider, mit der ein ebensolcher, respektvoller Umgang mit den Probanden gepflegt werden soll.

\section{Fazit}

Mithilfe der beschriebenen Qualitätskriterien sollen bereits vorliegende Erkenntnisse und etablierte Standards im Bereich der Behandlung von straffällig gewordenen Menschen für die ambulante Arbeit nutzbar gemacht werden. Sie weisen mit ihrer Spannbreite von scheinbar profanen Anforderungen an die räumliche Ausstattung einer forensischen Ambulanz bis hin zu hoch differenzierten Aspekten der Erfolgsmessung auf den großen Umfang der zur berücksichtigenden Faktoren zur Qualitätssicherung hin.

Mit einer konsequenten Umsetzung der Qualitätssicherungsmaßnahmen in den einzelnen Ambulanzen würde sich eine Datenbasis ergeben, mit der die Ambulanzen vergleichbar und somit die Daten über die Ambulanzen hinweg auswertbar werden. Mit einer unabhängigen, bundesweiten Evaluation der Ambulanzen - ähnlich der ersten Iststanderhebung durch die KrimZ (Gregório Hertz et al. 2017) könnten so wichtige Erkenntnisse für die ambulante therapeutische Versorgung von Probanden mit Sexual- und Gewaltstraftaten gewonnen werden. Die Unterschiede in der bundesweiten Umsetzung der forensischen Ambulanzen könnten im Sinne einer „evidence based practise“ eine Einschätzung erlauben, welche Interventionen im ambulanten Setting langfristig wirksam sind.

Interessenkonflikt C. Schwarze, T. Voß, O. Kliesch, A. Bauer, S. Braunisch, M.G. Feil, H. Fellmann, F. von Franqué, R. Freese, Y. Gretenkord, C. Huchzermeier, V. Jückstock, T. Klemm, H. KroonHeinzen, R. Martin, J. Pitzing, K. Wegner und M. Zisterer-Schick geben an, dass kein Interessenkonflikt besteht.

\section{Anhang}

Tab. 1 Übersicht Qualitätskriterien forensischer Ambulanzen des Strafvollzugs

\begin{tabular}{|c|c|}
\hline SQK 1 & Qualifikation der Mitarbeitenden/des Teams \\
\hline SQK 2 & Leitung und Fachaufsicht \\
\hline SQK 3 & Juristischer Beistand \\
\hline SQK 4 & Auslastung einer Ambulanz \\
\hline SQK 5: & Krisenmanagement \\
\hline SQK 6: & Betreuung nach Beendigung der Weisung \\
\hline SQK 7: & Kooperationsnetzwerk \\
\hline SQK 8: & Regionale Zuständigkeit/Erreichbarkeit \\
\hline SQK 9: & Ausstattung und Finanzierung \\
\hline SQK 10: & Existenz eines Behandlungskonzepts \\
\hline SQK 11: & Dokumentation und Qualitätssicherung \\
\hline PQK 1 & Qualitätskriterien im Verlauf der Behandlung \\
\hline PQK 1.1: & Transparenz der Behandlung und Aufklärungspflichten \\
\hline PQK 1.2: & Erfassung und Aktualisierung der Stammdaten \\
\hline PQK 1.3: & Forensisch-psychologische Diagnostik und Risikobeurteilung \\
\hline PQK 1.4: & Erarbeitung und Aktualisierung der Delikthypothese \\
\hline PQK 1.5: & Individuelle Behandlungsplanung \\
\hline PQK 1.6: & $\begin{array}{l}\text { Fallbezogene Zuständigkeitsklärung, Arbeit im multiprofessionellen } \\
\text { Team }\end{array}$ \\
\hline PQK 1.7: & Professionelle Vernetzung \\
\hline PQK 1.8: & Einbeziehung des persönlichen Umfeldes \\
\hline PQK 2 & Metaprinzipien der Prozessqualität \\
\hline PQK 2.1: & Evidenzbasierung, theoretische Fundierung und Werteorientierung \\
\hline PQK 2.2: & Kriterienorientierte Entscheidungen \\
\hline PQK 2.3: & $\begin{array}{l}\text { Regelung des Umgangs mit Offenbarungs- und Verschwiegenheits- } \\
\text { pflichten und -rechten }\end{array}$ \\
\hline PQK 2.4: & Mehraugenprinzip \\
\hline PQK 2.5: & Dokumentation \\
\hline PQK 2.6: & Verlaufsanalyse und Fehlerkultur \\
\hline EQK 1 & Direkt deliktbezogene Kriterien \\
\hline EQK 1.1: & Gewaltvermeidung \\
\hline EQK 1.1.1: & Reduktion einschlägiger Gewalthandlungen \\
\hline EQK 1.1.2: & $\begin{array}{l}\text { Reduktion nichteinschlägiger Gewalthandlungen, sonstiger Delin- } \\
\text { quenz und weiterer dissozialer Verhaltensweisen }\end{array}$ \\
\hline EQK 1.2: & Reduktion von Risiko \\
\hline EQK 1.2.1: & Reduktion der Risikokategorie \\
\hline EQK 1.2.2: & Reduktion der Risiko-Scores \\
\hline EQK 1.2.3: & Reduktion relevanter Risikofaktoren \\
\hline EQK 1.3: & Anbindung/Drop-out \\
\hline EQK 2 & Mittelbar deliktbezogene Kriterien \\
\hline EQK 2.1: & Ansprechbarkeit \\
\hline EQK 2.2: & Zufriedenheit der Probanden \\
\hline EQK 2.2.1: & Subjektive Zufriedenheit mit der Behandlung \\
\hline EQK 2.2.2: & Subjektive Lebenszufriedenheit \\
\hline EQK 2.3: & Compliance \\
\hline EQK 2.4: & Soziale Integration \\
\hline EQK 2.5: & Team \\
\hline
\end{tabular}

$S Q K$ Strukturqualitätskriterium, $P Q K$ Prozessqualitätskriterium, $E Q K$ Ergebnisqualitätskriterium 
Open Access Dieser Artikel wird unter der Creative Commons Namensnennung 4.0 International Lizenz (http://creativecommons.org/ licenses/by/4.0/deed.de) veröffentlicht, welche die Nutzung, Vervielfältigung, Bearbeitung, Verbreitung und Wiedergabe in jeglichem Medium und Format erlaubt, sofern Sie den/die ursprünglichen Autor(en) und die Quelle ordnungsgemäß nennen, einen Link zur Creative Commons Lizenz beifügen und angeben, ob Änderungen vorgenommen wurden.

\section{Literatur}

Andrews DA, Bonta J (2010) The psychology of criminal conduct, 5. Aufl. LexisNexis, New Providence

von Franqué F, Briken P (2017) Behandlung von Sexualdelinquenz. In: Büttner M (Hrsg) Sexualität und Trauma. Schattauer, Stuttgart

Freese R, Schmidt-Quernheim F (2014) Mindeststandards forensischer Nachsorge. Qualitätskriterien in forensisch-psychiatrischen Nachsorgeambulanzen. Forens Psychiatr Psychol Kriminol 8:191-198

Gregório Hertz P, Breiling L, Schwarze C, Klein R, Rettenberger M (2017) Extramurale Behandlung und Betreuung von Sexualstraftätern: Ergebnisse einer bundesweiten Umfrage zur NachsorgePraxis 2016. BM-Online, Bd. 13. KrimZ, Wiesbaden

Hart SD, Kropp PR, Laws DR (2003) The risk for sexual violence protocol (RSVP). Structured pofessional guidelines for assessing the risk of sexual violence. Mental Health, Law, \& Policy Institute of the Simon Fraser University, Pacific Psychological Assessment Cooperation and The British Columbia Institute against Familiy Violence, Vancouver

Kropp PR, Hart SD, Webster CD, Eaves D (1999) Manual for the spousal assault risk assessment guide, 3. Aufl. Multi-Health Systems, Toronto
Kropp PR, Hart SD, Lyon DR (2008) The Stalking Assessment and Management guidelines (SAM): user manual. ProActive ReSolutions, Vancouver

Sauter J, Voß T, Dahle K-P (2015) Wirksamkeit ambulanter Nachsorge bei Strafvollzugsentlassenen. Nervenarzt 86:571-578

Sauter J, Seewald K, Dahle K-P (2017) Wirksamkeit ambulanter forensischer Nachsorge - Was wissen wir wirklich? Übersichtsarbeit über den aktuellen Forschungsstand in der Bundesrepublik Deutschland. Bewährungshilfe Soz Strafr Kriminalpolit 64(2):146-161

Schwanengel F, Endres J (2016) Kriminaltherapeutische Straftäterbehandlung. Theoretische Modelle und praktische Umsetzungen. Forum Strafvollzug 65(3):158-162

Schwarze C, Freese R (2014) Ambulanzen des Straf- und Maßregelvollzugs. Was wir voneinander lernen können. 5. Treffen der Forensischen Nachsorgeambulanzen, Hamburg (Präsentation)

Voß T, Klemke K, Schneider-Njepel V, Kröber H-L (2015) Forensische Rehabilitation zuvor Sicherungsverwahrter in der Forensisch-Therapeutischen Ambulanz Berlin. Forens Psychiatr Psychol Kriminol 9:38-46

Wade DT, de Jong BA (2000) Recent advances in rehabilitation. Br Med J 320:1385-1388

Ward T, Gannon TA (2006) Rehabilitation, etiology, and self-regulation: The Good Lives Model of sexual offender treatment. Aggress Violent Behav 11:77-94

Ward T, Maruna S (2007) Rehabilitation: beyond the risk paradigm. Routledge, London

Webster CD, Douglas KS, Eaves D, Hart SD (1997) HCR-20. Assessing the risk for violence (version 2). Mental Health, Law, and Policy Institute, Simon Fraser University, Vancouver 